\title{
Buckling Analysis of Intermediately Supported Nanobeams via Strain Gradient Elasticity Theory
}

\author{
Mustafa Arda \\ Trakya University, Department of Mechanical Engineering, 22130, Edirne, TURKEY \\ ${ }^{*}$ E-mail address: mustafaarda@trakya.edu.tr \\ ORCID number of author: \\ 0000-0002-0314-3950
}

Received date: 17.12 .2020

Accepted date: 29.12 .2020

\begin{abstract}
Buckling of axially loaded cantilever nanobeams with intermediate support have been studied in the current study. Higher order size dependent strain gradient theory has been utilized to capture the scale effect in nano dimension. Minimum total potential energy formulation has been used in modeling of nanobeam. Approximate Ritz method has been applied to the energy formulation for obtaining critical buckling loads. Position of the intermediate support has been varied and its effect on the critical buckling load has been investigated in the analysis. Mode shapes in critical buckling loads have been shown for various intermediate support positions. Present results could be useful in design of carbon nanotube resonators.
\end{abstract}

Keywords: Nanobeam, Strain Gradient, Intermediate Support, Ritz Method.

\section{Introduction}

Carbon nanotubes (CNTs) have had an increasing popularity over the last three decades in academia and industry. Superior properties like thermal, electromagnetic, strength, etc. have enriched the possible usage areas of CNTs [1-3].

Statics and dynamics of nanoscale structures can be achieved with higher order size dependent continuum mechanics theories: strain gradient [4], stress gradient [5,6], couple stress[7], doublet mechanics [8] and peridynamics [9]. It has been presented in earlier works that classical elasticity theory is inadequate in the modelling of CNTs due to its size independent characteristics.

Basis of the higher order size dependent theories went back to a century ago. Cauchy [10], Voigt [11] and Cosserat brothers [12] had constituted the higher gradient elasticity theory. Kunin [13], Toupin [14], Mindlin [15], Kröner [16], Green and Rivlin [17] improved the higher order elasticity theories with including microstructural effects.

Eringen [18] proposed the nonlocal elasticity theory which is a stress gradient model. After Eringen, Aifantis and coworkers [19-22], proposed a higher order strain gradient elasticity theory for finite and infinitesimal deformations. Theories of Eringen and Aifantis are comparatively simple and includes less number of higher order gradient terms than previous 
ones. Higher order strain gradient models have been applied to the buckling problem of nano structures in several papers [23-32] . Over the last 20 years, higher order stress and strain driven continuum mechanics theories have been used in many studies [33-46].

In the present study, strain gradient nanobeam model has been developed for the buckling of axially loaded cantilever nanobeam with intermediate support. Higher order governing equation of motion for nanobeam have been obtained with minimum total potential energy formulation. Approximate Ritz Method has been used in the solution of the governing equation of motion. Effect of the position of intermediate support to the critical buckling load of nanobeam. Mode shapes at critical buckling loads for the first three modes have been depicted in various position of intermediate support. Differently from the previous studies, position of the intermediate support has been investigated in buckling case using strain gradient theory.

\section{Analysis}

A nanobeam of hollow tube with length $L$ is considered (Fig. 1). $x$ and $z$ axes define the axial length direction and transverse direction of nanobeam, respectively. $P$ is the external axial load and position of the intermediate support is defined as $\eta L$.

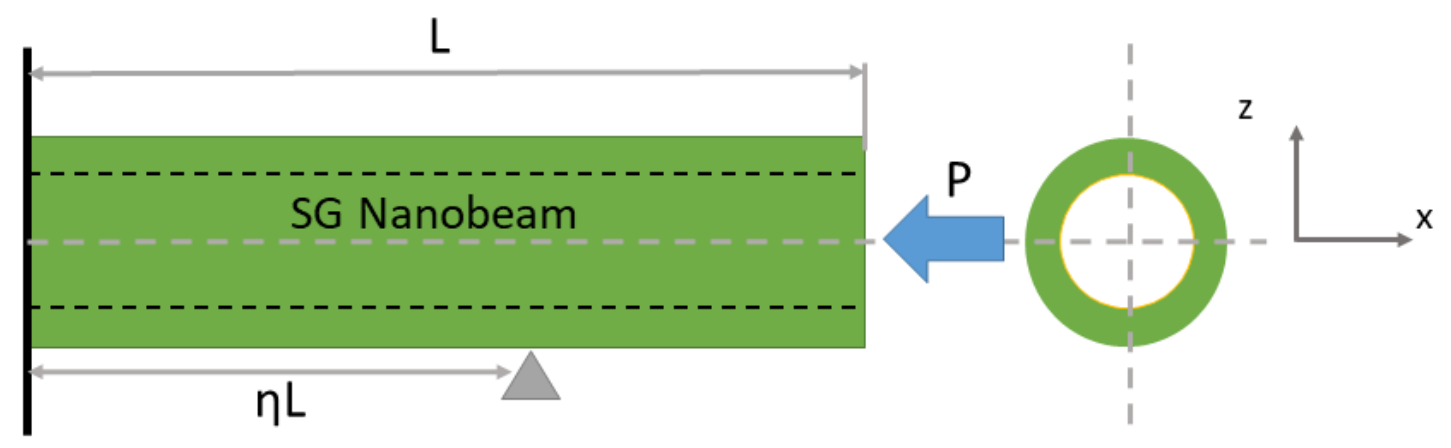

Fig. 1. Axially Loaded Nanobeam with Intermediate Support

\subsection{Strain Gradient Theory}

Refined form of the strain gradient elasticity theory can be interpreted for stress-strain relation as below [19-22]:

$$
\sigma_{i j}=\lambda \bar{\varepsilon}_{k k} \delta_{i j}+2 \mu \bar{\varepsilon}_{i j}, \quad \bar{\varepsilon}_{i j}=\varepsilon_{i j}-l \nabla^{2} \varepsilon_{i j}
$$

where $\sigma$ and $\varepsilon$ are the stress and strain tensors for elastic deformation respectively, $\nabla$ is the Laplacian, $\lambda$ and $\mu$ are the standard Lame constants and $l$ is the strain gradient parameter.

If the constituve equation is reformulated for one dimensional structures, the stress strain relation for the nanobeam can be obtained:

$$
\sigma_{x x}=\left(1-l \frac{\partial^{2}}{\partial x^{2}}\right) E \varepsilon_{x x}
$$

Total potential energies for the nanobeam can be defined as below:

$$
T=\frac{1}{2} \int_{0}^{L} P\left(\frac{\partial w}{\partial \mathrm{x}}\right)^{2} d x
$$




$$
U=\frac{1}{2} \int_{0}^{L} E I\left(\frac{\partial^{2} w}{\partial x^{2}}\right)^{2} d x+\frac{1}{2} \int_{0}^{L} l E I\left(\frac{\partial^{3} w}{\partial x^{3}}\right)^{2} d x
$$

where $I$ is moment of inertia for the nanobeam, $T$ defines the work done by external axial load and $U$ defines the potential energy of nanobeam. It should be noted that there is no kinetic energy in the present static buckling problem.

\subsection{Ritz method}

Analytical solution of the higher order governing equation of motions becomes complicated and time consuming with increasing number of boundary conditions and integration constants. Ritz method is a useful approximate variational method can be used in the solution of the mentioned problem [47-49]. Also discrete singular convolution method [50-53] and finite element modelling [54] can be used as an approximate solution.

Displacement function can be defined as in the below form for the Ritz method [55]:

$$
w(\bar{x})=\sum_{j=j_{0}}^{J} A_{j} \psi_{j}(\bar{x})
$$

where $\bar{x}$ is the dimensionless nanobeam length $\left(\bar{x}=\frac{x}{L}\right), A_{j}$ 's are the unknown coefficients and $\psi_{j}(\bar{x})$ is a function which satisfies geometric boundary conditions of the beam. Convergence of this function is satisfied if this function is mathematically complete set. To determine the critical buckling of nanobeam, next functional is defined:

$$
F=T_{\max }-U_{\max }
$$

This functional should be minimized with respect to unknown coefficients given in Eq. (5):

$$
\begin{gathered}
\frac{\partial F}{\partial A_{\bar{J}}}=0, \bar{J}=\bar{J}_{0}, \ldots, J \\
F=\frac{1}{2} \int_{0}^{1} P_{c r} \frac{\partial w}{\partial \bar{x}} \frac{\partial \bar{w}}{\partial \bar{x}} d \bar{x}-\frac{1}{2} \int_{0}^{1} \frac{\partial^{2} w}{\partial \bar{x}^{2}} \frac{\partial^{2} \bar{w}}{\partial \bar{x}^{2}} d \bar{x}-\frac{1}{2} \int_{0}^{1} \frac{l}{L^{2}} \frac{\partial^{3} w}{\partial \bar{x}^{3}} \frac{\partial^{3} \bar{w}}{\partial \bar{x}^{3}} d \bar{x}
\end{gathered}
$$

where $P_{c r}$ is the dimensionless critical buckling load of nanobeam and defined as below:

$$
P_{c r}=\frac{P L^{2}}{E I}
$$

Eq. (8) gives a total of $J \times J$ simultaneous, linear, homogeneous equations in an equal number of unknowns $A_{j}$. Those equations can be described as an eigen-value problem for critical buckling load. The mode shapes corresponding to any $P_{c r}$ is found by substituting that value into Eq. (7) and solving for the eigenvector components $A_{j} / A_{1}$. Inserting these components into Eq. (7) gives mode shape of nanobeam.

$\psi_{j}(\bar{x})$ polynomial can be assumed as below in general form.

$$
\psi_{j}=(\bar{x}-0)^{b_{1}}(\bar{x}-\eta)^{b_{2}}(\bar{x}-1)^{b_{3}}\left(\bar{x}^{j-1}\right)
$$


where $b_{1}, b_{2}$ and $b_{3}$ parameters define the boundary conditions and should be selected as $0,1,2$ for the free, simply supported and clamped boundary conditions, respectively. For the present clamped-simply supported-free nanobeam case, Eq. (10) turns into:

$$
\psi_{j}=(\bar{x})^{2}(\bar{x}-\eta)\left(\bar{x}^{j-1}\right)
$$

\section{Numerical Results}

Buckling analysis of the axially loaded nanobeam has been carried out for position of intermediate support and strain gradient parameter in this section. Analysis has been made independent from the material properties, except the nanobeam length which is assumed $5 \mathrm{~nm}$. Interested readers can look to previous paper [56] about selection of the length scale parameter.

Convergence of the Ritz method is seen in Table 1 for the first three critical buckling loads on local $(l=0)$ clamped-free and clamped-simply supported beams. Ritz method converges to literature works when $J$ is assumed as 7 .

Table 1. Validation of the Ritz Solution

\begin{tabular}{lllll}
\hline \multirow{2}{*}{$\begin{array}{l}\text { Mode } \\
\text { Number }\end{array}$} & \multicolumn{2}{l}{ Clamped-Free } & \multicolumn{3}{c}{$\begin{array}{l}\text { Clamped-Simply } \\
\text { Supported }\end{array}$} \\
\cline { 2 - 5 } & $P_{c r}=\frac{\pi^{2}}{4}$ & Ritz Method & $P_{c r}=\frac{\pi^{2}}{0.7^{2}}$ & Ritz Method \\
\hline 1 & 2.4674 & 2.4674 & 20.1907 & 20.1907 \\
\hline 2 & 22.2066 & 22.2066 & 59.6795 & 59.6803 \\
\hline 3 & 61.6850 & 61.7017 & 118.9000 & 119.0870 \\
\hline
\end{tabular}

In Table 2, strain gradient parameter effect on critical buckling load can be seen. Strain gradient theory exhibits stiffening effect on structure. Growing rigidity increases the critical buckling load. Position of the intermediate support should be investigated with using both Table 2 and Figure 2. Critical buckling load increases when intermediate support approaches to the free end at the first mode. On the other hand, second and third mode critical buckling loads firstly increase, then goes constant little bit and decreases after that. Third mode buckling load also initially increases than start to decrease. Cause of this behavior should be related with the nodal points of mode shapes which can be seen in Figures 3 and 4. If the intermediate support is placed after a nodal point, nanobeam can buckle more easily.

Table 2. Strain Gradient Theory Effect on Critical Buckling Loads

\begin{tabular}{lllllll}
\hline \multirow{2}{*}{$\begin{array}{l}\text { Node } \\
\text { Number }\end{array}$} & \multicolumn{2}{l}{ Local Theory $(l=0)$} & \multicolumn{4}{c}{$\begin{array}{l}\text { Strain Gradient Theory } \\
\left(l=0.1 \mathrm{~nm}^{2}\right)\end{array}$} \\
\cline { 2 - 7 } & $\eta=0.1$ & $\eta=0.5$ & $\eta=1$ & $\eta=0.1$ & $\eta=0.5$ & $\eta=1$ \\
\hline 1 & 2.8912 & 6.2714 & 20.1907 & 3.2152 & 6.6923 & 21.7518 \\
\hline 2 & 26.0184 & 52.2509 & 59.6803 & 31.0665 & 62.4753 & 72.4724 \\
\hline 3 & 72.2408 & 120.7560 & 119.0870 & 97.9405 & 171.5440 & 169.417 \\
\hline
\end{tabular}



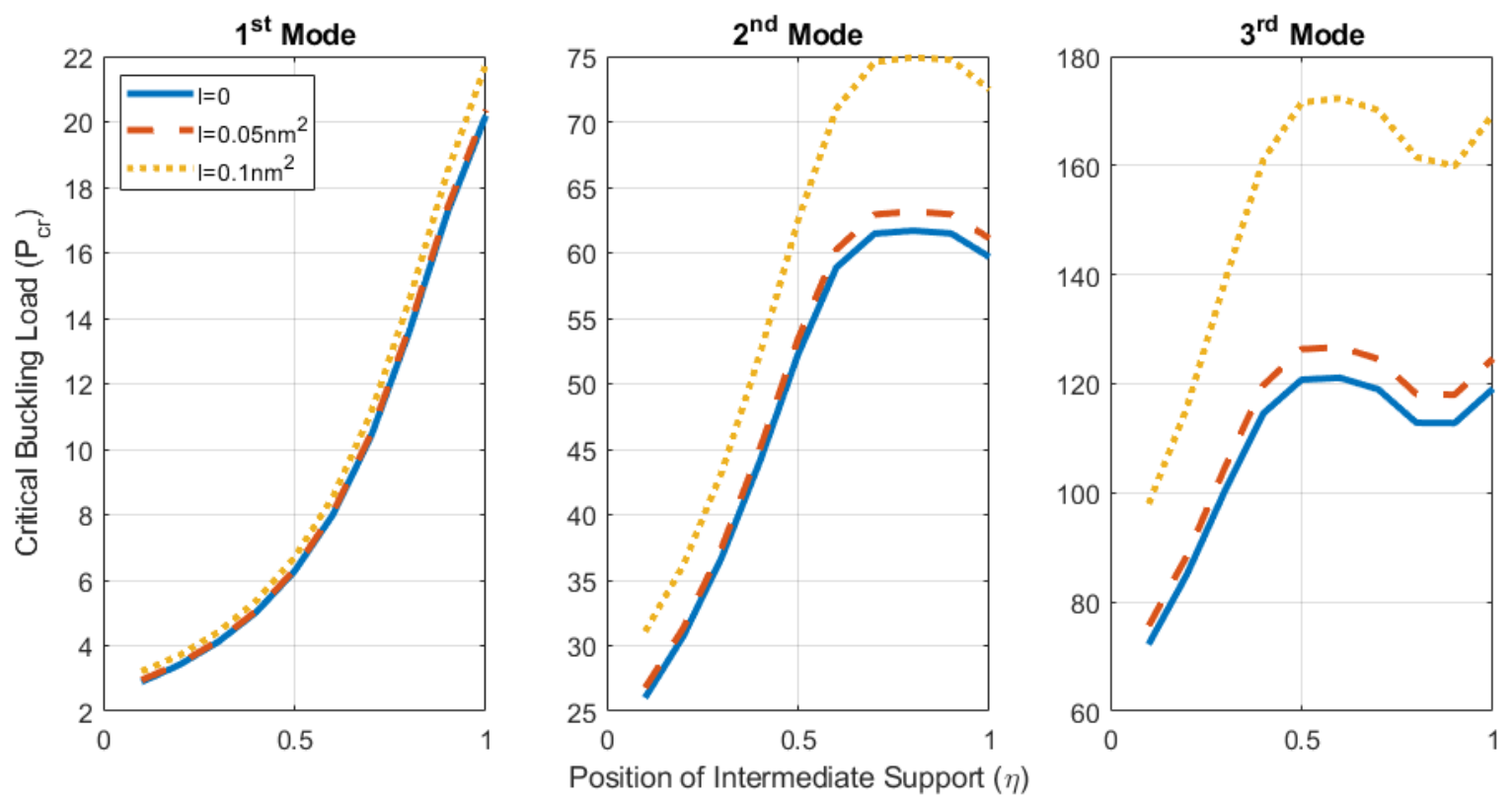

Fig. 2. Variation of Critical Buckling Load with Position of Intermediate Support

In Figures 3 and 4, mode shapes of nanobeam at the first three critical buckling loads are seen. Increasing critical buckling load enhances the relative amplitude of displacements. Position of the intermediate support has an important effect on mode shapes. Also, strain gradient theory increases the amplitudes in mode shapes as a result of increasing critical buckling load.
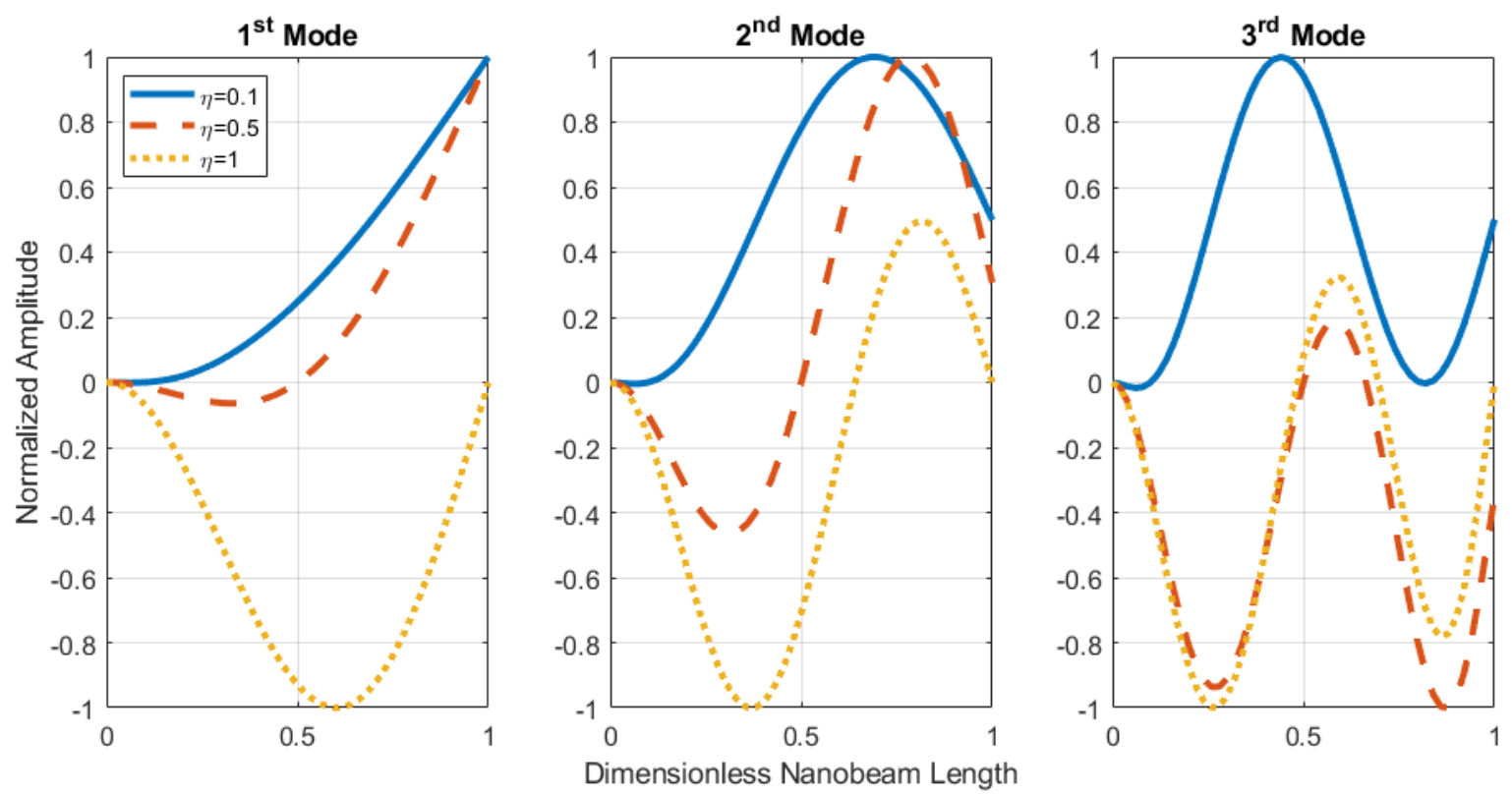

Fig. 3. Mode Shapes of Nanobeam at Various Intermediate Support Positions $(l=0)$ 

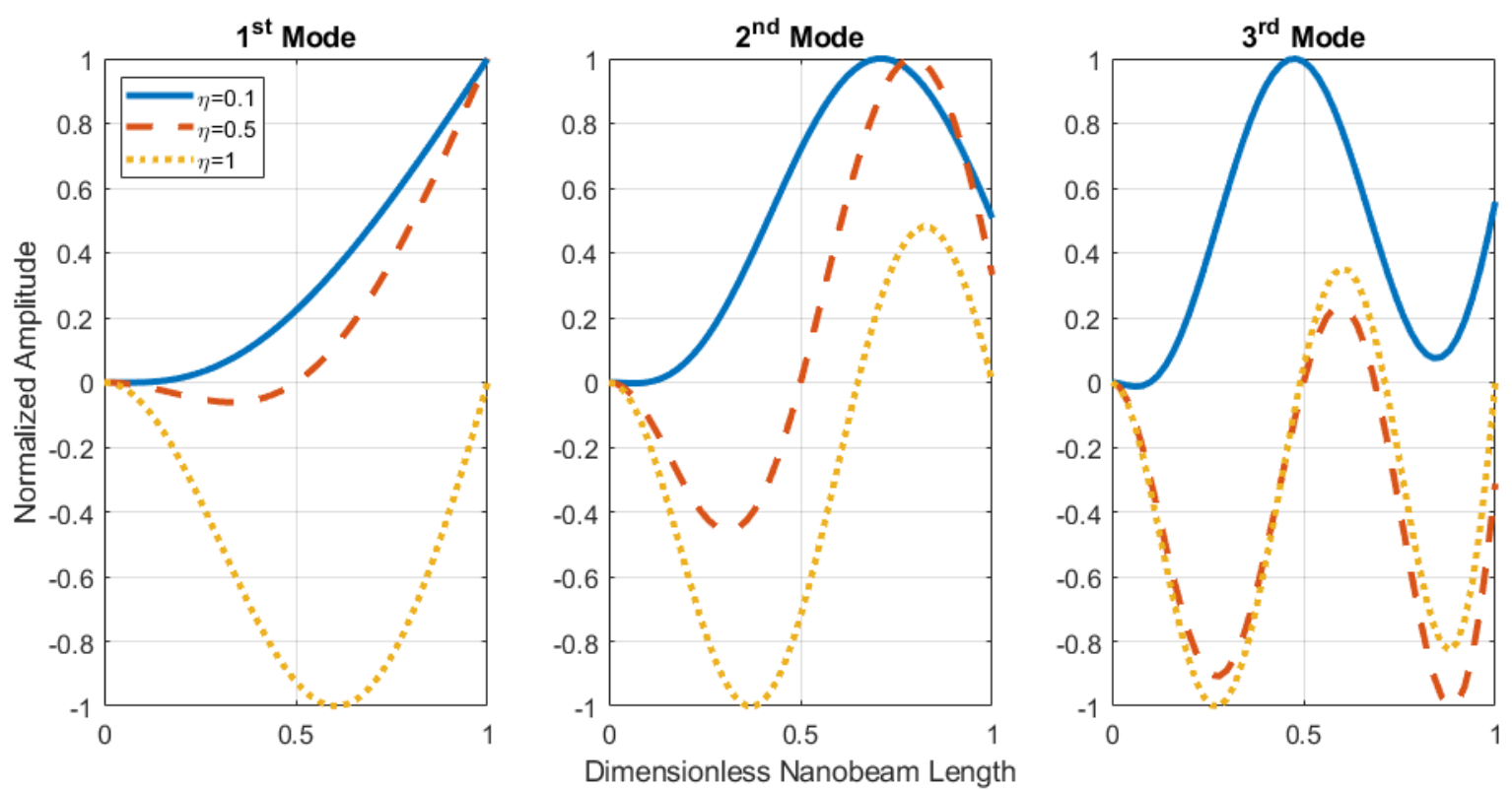

Fig. 4. Mode Shapes of Nanobeam at Various Intermediate Support Positions $\left(l=0.1 \mathrm{~nm}^{2}\right)$

\section{Conclusion}

Present study has been investigated the buckling of axially loaded clamped-simply supportedfree nanobeams with using strain gradient theory. Minimum total potential energy formulation has been applied to the nanobeam to obtain the static equilibrium equation. Ritz method has been used on the energy formulation for obtaining of critical buckling load. Effects of the position of the intermediate support and strain gradient parameter to the critical buckling load has been investigated. Mode shapes in critical buckling loads have been depicted for local and strain gradient models in various intermediate support positions.

Strain gradient model increases the critical buckling load for nanobeam and normalized amplitudes with the stiffening effect. Position of the intermediate support increase or decrease the critical buckling load depending on the nodal points of adjacent mode numbers.

Present results could be useful in design of carbon nanotube resonators.

\section{References}

[1] Ajiki H., Ando T., Energy Bands of Carbon Nanotubes in Magnetic Fields, Journal of the Physical Society of Japan, 65, 505-14, 1996. doi:10.1143/JPSJ.65.505

[2] Craighead H.G., Nanoelectromechanical Systems, Science, 290, 1532-5, 2000. doi:10.1126/science.290.5496.1532

[3] Huang X.M.H., Zorman C.A., Mehregany M., Roukes M.L., Nanoelectromechanical systems: Nanodevice motion at microwave frequencies, Nature, 421, 496-496, 2003. doi:10.1038/421496a

[4] Ghorbanpour Arani A., Shokravi M., Vibration response of visco-elastically coupled double-layered visco-elastic graphene sheet systems subjected to magnetic field via strain 
gradient theory considering surface stress effects, Proceedings of the Institution of Mechanical Engineers, Part N: Journal of Nanoengineering and Nanosystems, 229, 18090, 2015. doi:10.1177/1740349914529102

[5] Arda M., Aydogdu M., Torsional statics and dynamics of nanotubes embedded in an elastic medium, Composite Structures, 114, 80-91, 2014. doi:10.1016/j.compstruct.2014.03.053

[6] Li C., Li S., Zhu Z., Prediction of mechanical properties of microstructures through a nonlocal stress field theory, Proceedings of the Institution of Mechanical Engineers, Part N: Journal of Nanoengineering and Nanosystems, 229, 50-4, 2015. doi:10.1177/1740349913519437

[7] Kumar M., Reddy G.J., Kumar N.N., Bég O.A., Computational study of unsteady couple stress magnetic nanofluid flow from a stretching sheet with Ohmic dissipation, Proceedings of the Institution of Mechanical Engineers, Part N: Journal of Nanomaterials, Nanoengineering and Nanosystems, 233, 49-63, 2019. doi:10.1177/2397791419843730

[8] Gul U., Aydogdu M., Gaygusuzoglu G., Axial dynamics of a nanorod embedded in an elastic medium using doublet mechanics, Composite Structures, 160, 1268-78, 2017. doi:10.1016/j.compstruct.2016.11.023

[9] Oterkus E., Diyaroglu C., Zhu N., Oterkus S., Madenci E., Utilization of Peridynamic Theory for Modeling at the Nano-Scale, 2015, p. 1-16. doi:10.1007/978-3-319-21194-7_1

[10]Cauchy A.-L., Mémoire sur les systèmes isotropes de points matériels. Oeuvres complètes, Cambridge University Press, 1882. doi:10.1017/CBO9780511702280.023

[11]Voigt W., Theoretische Studien über die Elasticitätsverhältnisse der Krystalle, Abhandlungen der Königlichen Gesellschaft der Wissenschaften in Göttingen, 34, 3-52, 1887

[12] Cosserat E., Cosserat F., Theorie des corps dédormables. A. Hermann et fils, 1909

[13] Kunin I.A., Elastic Media with Microstructure I. vol. 26. Springer Berlin Heidelberg, 1982. doi:10.1007/978-3-642-81748-9

[14] Toupin R.A., Theories of elasticity with couple-stress, Archive for Rational Mechanics and Analysis, 17, 85-112, 1964. doi:10.1007/BF00253050

[15] Mindlin R.D., Micro-structure in linear elasticity, Archive for Rational Mechanics and Analysis, 16, 51-78, 1964. doi:10.1007/BF00248490

[16]Kröner E., Elasticity theory of materials with long range cohesive forces, International Journal of Solids and Structures, 3, 731-42, 1967. doi:10.1016/0020-7683(67)90049-2

[17] Green A.E., Rivlin R.S., Multipolar continuum mechanics, Archive for Rational Mechanics and Analysis, 17, 113-47, 1964. doi:10.1007/BF00253051

[18] Eringen A.C., Edelen D.G.B., On nonlocal elasticity, International Journal of Engineering Science, 10, 233-48, 1972. doi:10.1016/0020-7225(72)90039-0

[19]Ru C.Q., Aifantis E.C., A simple approach to solve boundary-value problems in gradient elasticity, Acta Mechanica, 101, 59-68, 1993. doi:10.1007/BF01175597

[20]Altan B.S., Aifantis E.C., On Some Aspects in the Special Theory of Gradient Elasticity, Journal of the Mechanical Behavior of Materials, 8, 1997. doi:10.1515/JMBM.1997.8.3.231 
[21]Aifantis E.C., Strain gradient interpretation of size effects, International Journal of Fracture, 95, 299-314, 1999

[22] Aifantis E.C., Higher Order Gradients and Self-Organization at Nano, Micro, and Macro Scales, Materials Science Forum, 123-125, 553-66, 1993. doi:10.4028/www.scientific.net/msf.123-125.553

[23]Akgöz B., Civalek Ö., Strain gradient elasticity and modified couple stress models for buckling analysis of axially loaded micro-scaled beams, International Journal of Engineering Science, 49, 1268-80, 2011. doi:10.1016/j.ijengsci.2010.12.009

[24]Akgöz B., Civalek Ö., Buckling analysis of functionally graded microbeams based on the strain gradient theory, Acta Mechanica, 224, 2185-201, 2013. doi:10.1007/s00707-013$0883-5$

[25]Akgöz B., Civalek Ö., A new trigonometric beam model for buckling of strain gradient microbeams, International Journal of Mechanical Sciences, 81, 88-94, 2014. doi:10.1016/j.ijmecsci.2014.02.013

[26]Mercan K., Civalek Ö., A Simple Buckling Analysis Of Aorta Artery, International Journal Of Engineering \& Applied Sciences, 7, 34-34, 2015. doi:10.24107/ijeas.251256

[27]Demir Ç., Mercan K., Civalek O., Determination of critical buckling loads of isotropic, FGM and laminated truncated conical panel, Composites Part B: Engineering, 94, 1-10, 2016. doi:10.1016/j.compositesb.2016.03.031

[28]Mercan K., Civalek Ö., Buckling Analysis of Silicon Carbide Nanotubes (SiCNTs), International Journal Of Engineering \& Applied Sciences, 8, 101-101, 2016. doi:10.24107/ijeas. 252148

[29] Arda M., Aydogdu M., Buckling of Eccentrically Loaded Carbon Nanotubes, Solid State Phenomena, 267, 151-6, 2017. doi:10.4028/www.scientific.net/SSP.267.151

[30]Mercan K., Civalek Ö., Buckling analysis of Silicon carbide nanotubes (SiCNTs) with surface effect and nonlocal elasticity using the method of HDQ, Composites Part B: Engineering, 114, 34-45, 2017. doi:10.1016/j.compositesb.2017.01.067

[31] Mercan K., Civalek Ö., Comparison of small scale effect theories for buckling analysis of nanobeams, International Journal Of Engineering \& Applied Sciences, 9, 87-97, 2017. doi:10.24107/ijeas.340958

[32]Mercan K., Numanoglu H.M., Akgöz B., Demir C., Civalek., Higher-order continuum theories for buckling response of silicon carbide nanowires (SiCNWs) on elastic matrix, Archive of Applied Mechanics, 87, 1797-814, 2017. doi:10.1007/s00419-017-1288-z

[33] Civalek Ö., Geometrically nonlinear dynamic and static analysis of shallow spherical shell resting on two-parameters elastic foundations, International Journal of Pressure Vessels and Piping, 113, 1-9, 2014. doi:10.1016/j.ijpvp.2013.10.014

[34] Arda M., Aydogdu M., Analysis of Free Torsional Vibration in Carbon Nanotubes Embedded in a Viscoelastic Medium, Advances in Science and Technology Research Journal, 9, 28-33, 2015. doi:10.12913/22998624/2361

[35]Ebrahimi F., Barati M.R., Civalek Ö., Application of Chebyshev-Ritz method for static stability and vibration analysis of nonlocal microstructure-dependent nanostructures, Engineering with Computers, 36, 953-64, 2020. doi:10.1007/s00366-019-00742-z 
[36] AlSaid-Alwan H.H.S., Avcar M., AlSaid-Alwan H.H.S., Avcar M., Analytical solution of free vibration of FG beam utilizing different types of beam theories: A comparative study, Computers and Concrete, 26, 285, 2020. doi:10.12989/CAC.2020.26.3.285

[37]Zhang J.S.Y.M.O., Analysis of orthotropic plates by the two-dimensional generalized FIT method, Computers and Concrete, 26, 421-7, 2020. doi:10.12989/CAC.2020.26.5.421

[38] Hadji L., Avcar M., Free Vibration Analysis of FG Porous Sandwich Plates under Various Boundary Conditions, J Appl Comput Mech, 0, 1-15, 2020. doi:10.22055/JACM.2020.35328.2628

[39] Arda M., Aydogdu M., Bending of CNTs Under The Partial Uniform Load, International Journal Of Engineering \& Applied Sciences, 8, 21-21, 2016. doi:10.24107/ijeas. 252142

[40]Arda M., Aydogdu M., Longitudinal Vibration of CNTs Viscously Damped in Span, International Journal Of Engineering \& Applied Sciences, 9, 22-22, 2017. doi:10.24107/ijeas.305348

[41]Mercan K., Civalek Ö., What is The Correct Mechanical Model of Aorta Artery, International Journal Of Engineering \& Applied Sciences, 9, 138-138, 2017. doi:10.24107/ijeas.322526

[42] Akgöz B., Civalek Ö., A size-dependent beam model for stability of axially loaded carbon nanotubes surrounded by Pasternak elastic foundation, Composite Structures, 176, 102838, 2017. doi:10.1016/j.compstruct.2017.06.039

[43] Arda M., Vibration Analysis of an Axially Loaded Viscoelastic Nanobeam, International Journal Of Engineering \& Applied Sciences, 10, 252-63, 2018. doi:10.24107/ijeas.468769

[44] Arda M., Aydogdu M., Dynamic stability of harmonically excited nanobeams including axial inertia, JVC/Journal of Vibration and Control, 25, 820-33, 2019. doi: $10.1177 / 1077546318802430$

[45] Arda M., Aydogdu M., Torsional dynamics of coaxial nanotubes with different lengths in viscoelastic medium, Microsystem Technologies, 25, 3943-57, 2019. doi:10.1007/s00542019-04446-8

[46]Jalaei M.H., Civalek., On dynamic instability of magnetically embedded viscoelastic porous FG nanobeam, International Journal of Engineering Science, 143, 14-32, 2019. doi:10.1016/j.ijengsci.2019.06.013

[47] Aydogdu M., Arda M., Filiz S., Vibration of axially functionally graded nano rods and beams with a variable nonlocal parameter, Advances in Nano Research, 6, 257-78, 2018. doi:10.12989/anr.2018.6.3.257

[48] Arda M., Aydogdu M., Vibration analysis of carbon nanotube mass sensors considering both inertia and stiffness of the detected mass, Mechanics Based Design of Structures and Machines, 0, 1-17, 2020. doi:10.1080/15397734.2020.1728548

[49] Arda M., Axial dynamics of functionally graded Rayleigh-Bishop nanorods, Microsystem Technologies, 2, 2020. doi:10.1007/s00542-020-04950-2

[50]Civalek O., Yavas A., Large Deflection Static Analysis of Rectangular Plates On Two Parameter Elastic Foundations, International Journal of Science and Technology, 1, 4350,2006

[51]Civalek Ö., Kiracioglu O., Free vibration analysis of Timoshenko beams by DSC method, 
International Journal for Numerical Methods in Biomedical Engineering, 26, 1890-8, 2010. doi:10.1002/cnm.1279

[52] Mercan K., Demir Ç., Civalek Ö., Vibration analysis of FG cylindrical shells with powerlaw index using discrete singular convolution technique, Curved and Layered Structures, 3, 82-90, 2016. doi:10.1515/cls-2016-0007

[53]Civalek Ö., Avcar M., Free vibration and buckling analyses of CNT reinforced laminated non-rectangular plates by discrete singular convolution method, Engineering with Computers, 1-33, 2020. doi:10.1007/s00366-020-01168-8

[54]Civalek Ö., Uzun B., Yaylı M.Ö., Akgöz B., Size-dependent transverse and longitudinal vibrations of embedded carbon and silica carbide nanotubes by nonlocal finite element method, European Physical Journal Plus, 135, 381, 2020. doi:10.1140/epjp/s13360-02000385-w

[55] Wright E.M., Kantorovich L. V., Krylov V.I., Benster C.D., Approximate Methods of Higher Analysis, The Mathematical Gazette, 44, 145, 1960. doi:10.2307/3612589

[56] Arda M., Evaluation of optimum length scale parameters in longitudinal wave propagation on nonlocal strain gradient carbon nanotubes by lattice dynamics, Mechanics Based Design of Structures and Machines, 1-24, 2020. doi:10.1080/15397734.2020.1835488 